\title{
Primary hepatic extranodal marginal zone B-cell lymphoma treated by surgical resection: $A$ case report
}

\begin{abstract}
Primary hepatic extranodal marginal zone B-cell lymphoma is extremely rare and remains an enigma with regard to clinicopathological features. Radiologic findings of primary hepatic extranodal marginal zone B-cell lymphoma are heterogenous. Therefore, the diagnosis is typically made by biopsy or after surgical resection. Due to a lack of evidence, there are no established standard therapeutic protocols or guidelines for this disease. Fortunately, these tumors tend to be indolent and respond well to various treatment modalities. Here, we report the case of a 72-year-old woman who was incidentally identified to have a solitary hepatic mass of $2.2 \mathrm{~cm}$ in diameter. The mass was found to be a primary hepatic extranodal marginal zone B-cell lymphoma after surgical resection with suspected hepatocellular carcinoma. The patient did not undergo postoperative chemotherapy. There was no evidence of recurrence after 8 months.
\end{abstract}

Volume 4 Issue 2 - 2018

Seo young Choi,' Jin Woo Lee, ' Sung Hoon

Choi,' Dae Jung Kim, ${ }^{2}$ and Gwangil Kim,

'Department of Surgery, CHA University, South Korea

${ }^{2}$ Radiology, CHA University, South Korea

${ }^{3}$ Pathology, CHA University, South Korea

\begin{abstract}
Correspondence: Sung Hoon Choi, Department of Surgery, CHA Bundang Medical Center, CHA University, Cancer Research Building \#524, 59 Yatap-ro, Bundang-gu, Seongnamsi, Gyeonggi-do 13496, South Korea, Tel 01030452 675, Fax 82317801824,Email feel415@cha.ac.kr
\end{abstract}

Received: March 02, 2018 | Published: April 16, 2018
Abbreviations: NHL, non-hodgkin lymphoma; CEA, carcinoembryonic antigen; MRI, magnetic resonance image; ERCP, endoscopic retrograde cholangiopancreraticography; MALT, mucosaassociated lymphoid tissue; CEA, carcinoembryonic antigen; CT, computed tomography; AFP, alpha fetoprotein

\section{Introduction}

There are many complicated subtypes of non-Hodgkin lymphoma (NHL). The World Health Organization and the Revised European American Lymphoma Classification subclassified NHL according to clinicopathological and characteristic histological features. ${ }^{1,2}$ One subtype of NHL is marginal zone lymphoma, which is relatively rare and indolent. Extranodal marginal zone B-cell lymphoma (also known as mucosa-associated lymphoid tissue (MALT) lymphoma) is the most common type of marginal zone lymphoma, accounting for 7-8\% of all NHLs. ${ }^{3}$ These rare extranodal marginal zones B-cell lymphomas most commonly originate in the stomach. However, these tumors can also arise in any extranodal site, including the skin, conjunctiva, salivary gland, thyroid, lung, breast, gallbladder, and liver. ${ }^{4,5}$

Primary hepatic lymphoma is extremely rare, with no clearly defined clinicopathological features. Based on a literature review by Dong at al. ${ }^{3}$ in 2017 , only 67 cases of primary hepatic lymphoma have been reported worldwide. We report a case of primary hepatic extranodal marginal zone B-cell lymphoma (or MALT lymphoma) diagnosed after surgical resection, with suspected concurrent hepatocellular carcinoma.

\section{Case report}

A 72-year-old woman was incidentally found to have a $2.2 \mathrm{~cm}$ solitary tumor in the right inferior segments on computed tomography (CT). The patient had undergone endoscopic retrograde cholangiopancreraticography (ERCP) one year prior for treatment of cholangitis due to common bile ducts stones. Several gallbladder stones were identified. Cholecystectomy was recommended at that time, but the patient deferred surgery. A liver mass was subsequently identified on follow-up CT, in addition to several gallstones. The patient was asymptomatic. She had no history of alcohol abuse or hepatitis B or hepatitis $\mathrm{C}$ viral infection. Blood cell counts and liver enzymes were unremarkable. Alpha fetoprotein (AFP), carbohydrate antigen 19-9 (CA19-9) and carcinoembryonic antigen (CEA) were also within their normal reference ranges. On contrast-enhanced CT scan (non-dynamic CT), the lesion in the S5/6 segment demonstrated heterogeneous enhancement and was described as cholangiocellular carcinoma (Figure 1). Liver magnetic resonance image (MRI) revealed a T1 hypointensity and T2 hyperintensity round well-defined mass. This mass was slightly enhanced in the arterial phase and subsequently washed-out in the portal phase (Figure 2). Hepatocellular carcinoma was highly suspected based on these MRI findings.

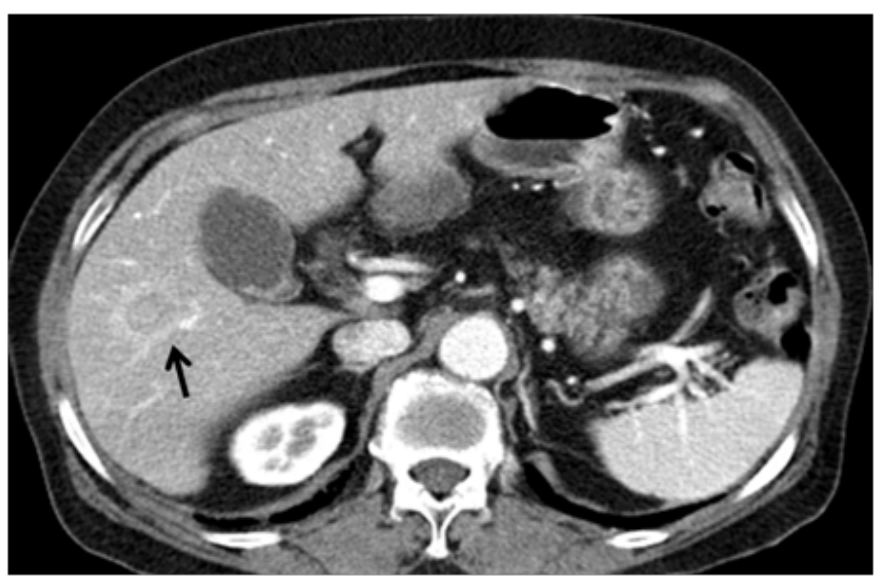

Figure I Contrast-enhanced CT scan.The lesion in segment S5/6 demonstrates heterogenous enhancement and is described as cholangiocellular carcinoma. 


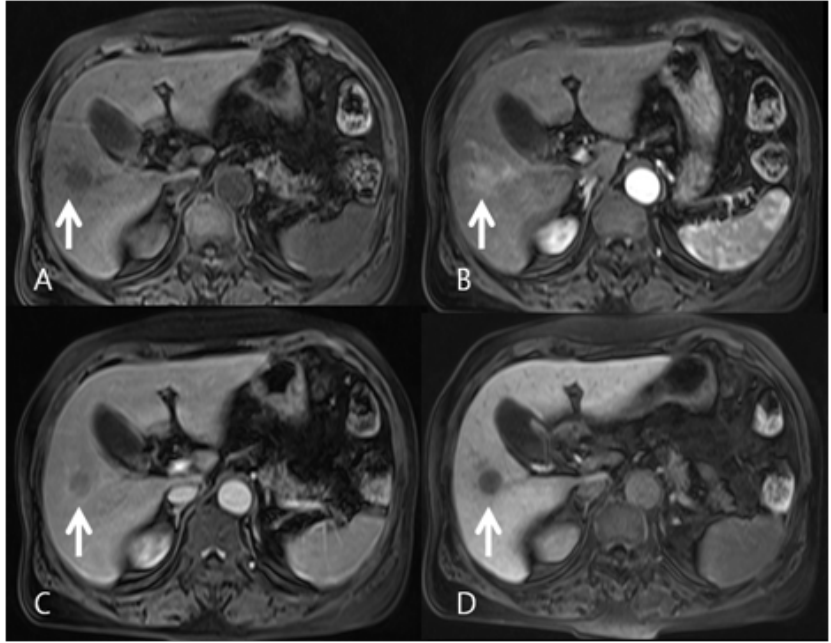

Figure 2 Liver magnetic resonance image (MRI). (A) Round low signal mass in precontrast image was slightly enhanced in the arterial phase (B) and subsequently washed-out in the portal phase (C). The mass shows a defect on the 20 minute delay phase of primovist (D). Hepatocellular carcinoma was highly suspected based on these MRI findings.

We do not usually perform preoperative needle biopsies in cases that are suspicious for malignant primary hepatic tumor or liver metastasis of colon cancer (if the cases are operable). The lesion was surgically removed by laparoscopic right inferior bisegmentectomy (S5/6). Pathology results revealed extranodal marginal zone B-cell lymphoma. The gross specimen was $2.2 \mathrm{~cm}$ in diameter with a "fish flesh" appearance (Figure 3). Subsequent microscopic examinations revealed dense lymphocyte infiltration of the proliferative marginal zone with destructive follicular structures (Figure 4A). There was also infiltration of lymphoid cells in the adjacent portal tract (Figure 4B). Immunochemistry studies were positive for CD20 and CD138, but negative for CD3, CD5, CD23, and S-100 (Figures 4C) (Figure $4 \mathrm{D})$. Ultimately, the diagnosis of indolent extranodal marginal zone B-cell lymphoma was made based on the histologic findings of three experienced pathologists. The patient did not undergo postoperative chemotherapy. Instead, she was followed periodically with surveillance follow-up CT scans to detect progression or lymphomarelated metastasis. There was no evidence of relapse over 8 months.

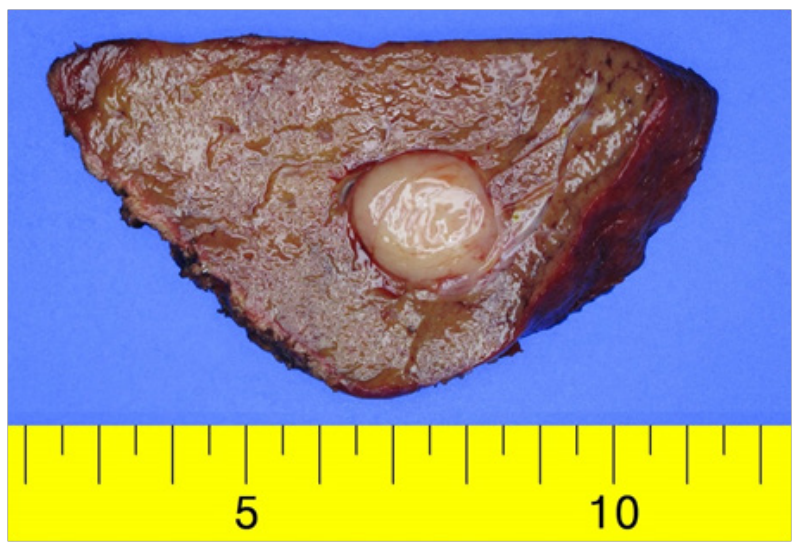

Figure 3 The gross specimen demonstrates a $2.2 \mathrm{~cm}$ mass (in diameter) with a "fish flesh" appearance.

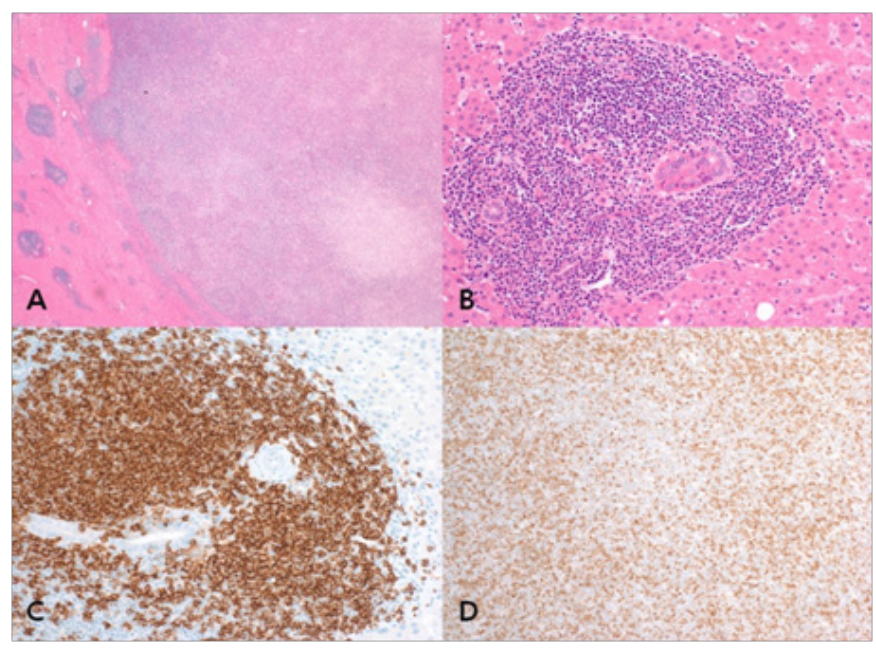

Figure 4 Characteristics of the histological examination. The lesion consists of dense lymphocyte infiltration of the proliferative marginal zone with destructive follicular structures (A) Hematoxylin and Eosin, HE XI2. Infiltration of lymphoid cells in the adjacent portal tract (B) HE X200. Immunochemistry studies were positive for CD20 in the adjacent portal tract (C) Immunohistochemical staining, IHC X40 and negative for CD3 (D) IHC $\times 100$.

\section{Discussion}

Although extranodal marginal zone B-cell lymphoma (or MALT lymphoma) can occur at any site (even those normally devoid of lymphoid tissue), primary hepatic lymphomas remain very rare. Therefore, this case report shares our experience with this rare disease, as well as a literature review.

A recent literature review ${ }^{3}$ identified only 67 cases of primary hepatic MALT lymphoma in the English literature. Preoperative radiographic findings tend to be heterogeneous; the diagnosis is most often made by biopsy or after surgical resection. ${ }^{6,7}$ These tumors commonly present with a solitary mass (74\% of cases) $0.7-9.0 \mathrm{~cm}$ in size. ${ }^{3,7}$ On contrast-enhanced CT scan, primary hepatic MALT lymphoma has a variable pattern, including no enhancement, faint enhancement, or peripheral enhancement in the early arterial phase. ${ }^{3,8}$ Literature MRI findings of primary hepatic MALT lymphoma are similar to those in our case, including hypointensity on T1-weighted images, moderate hyperintensity on T2-weighted images, and a slight and peripheral enhancement pattern. ${ }^{9}$ Therefore, in most cases, the preoperative diagnosis was non-specific, prompting biopsy $(8,9)$ or surgery if there was suspicion of cholangiocellular carcinoma ${ }^{3,7}$ or hepatocellular carcinoma. ${ }^{8}$

The etiology of hepatic primary lymphoma is not fully understood. There is literature to suggest a strong relationship between Helicobacter pylori-associated chronic gastritis and gastric MALT lymphoma. ${ }^{10}$ In most hepatic primary lymphoma patients, tumors are found incidentally. However, many patients also have concomitant disease, including chronic inflammatory liver disease (chronic hepatitis or liver cirrhosis related with hepatitis B or C virus, biliary disease, and so on), malignant tumors (colon cancer, gastric cancer, breast cancer, and so on), autoimmune disease (Sjogren syndrome, rheumatoid arthritis, Hashimoto thyroiditis, and so on), and Helicobacter pylori infection. ${ }^{3,7,8}$ The presence of such comorbidities 
suggests an association between chronic inflammatory conditions and primary hepatic MALT lymphoma. Regardless, further investigation is needed to investigate this hypothesis. In our case, it was unclear whether the patient's history of cholangitis was related to her cancer diagnosis.

There are no standard therapeutic protocols or guidelines for primary hepatic lymphoma due to its rarity and a lack of evidence. Patients have been treated with surgical resection, chemotherapy, rituximab (anti-CD20 monoclonal antibody), radiotherapy, and liver transplantation., ${ }^{3,7}$ In most cases, there is good treatment response. However, recurrences have been observed in patients who only underwent local treatments, including surgery or radiation therapy. ${ }^{7}$ However, these recurrences occurred 40-96 months after treatment, and few of these long-term follow-up patients had received chemotherapy. Therefore, it is difficult to conclude whether chemotherapy is indispensable for such patients. In the case presented here, the patient was older with low-grade lymphoma by pathology; therefore, a clinical decision was made to follow her conservatively (rather than to treat with chemotherapy).

\section{Conclusion}

Primary hepatic extranodal marginal zone B-cell lymphoma is a very rare disease with nonspecific clinical and radiographic features. In addition, there are no established treatment guidelines for primary hepatic extranodal marginal zone B-cell lymphoma. Fortunately, these tumors demonstrate indolent behavior and have a good response to various treatment modalities. Surgical resection can be a good option for both the diagnosis and treatment of patients with resectable disease. Despite having a good prognosis, late recurrence of primary hepatic lymphoma has been reported. Therefore, long-term follow-up is important.

\section{Acknowledgements}

All authors contributed towards data analysis, drafting and revising the paper and agree to be accountable for all aspects of the work.

\section{Conflict of interest}

There is no conflict of interest.

\section{References}

1. Harris NL, Jaffe ES, Diebold J, et al. World Health Organization classification of neoplastic diseases of the hematopoietic and lymphoid tissues: report of the Clinical Advisory Committee meeting, Airlie House, Virginia, November, 1997. Ann Oncol. 1999;10(12):1419-1432.

2. Harris NL, Jaffe ES, Stein H, et al. A revised European-American classification of lymphoid neoplasms: a proposal from the International Lymphoma Study Group. Blood. 1994;84(5):1361-1392.

3. Dong S, Chen L, Chen Y, et al. Primary hepatic extranodal marginal zone B-cell lymphoma of mucosa-associated lymphoid tissue type: A case report and literature review. Medicine (Baltimore). 2017;96(13):e6305.

4. Zucca E, Conconi A, Pedrinis E, et al. Nongastric marginal zone B-cell lymphoma of mucosa-associated lymphoid tissue. Blood. 2003;101(7):2489-2495.

5. Karia M, Mitsopoulos G, Patel K, et al. Primary Gallbladder Lymphoma in a Male Patient with No Risk Factors Detected Incidentally by CT Colonography. Case Rep Surg. 2015;2015:813708.

6. Obiorah IE, Johnson L, Ozdemirli M. Primary mucosa-associated lymphoid tissue lymphoma of the liver: A report of two cases and review of the literature. World J Hepatol. 2017;9(3):155-160.

7. Nagata S, Harimoto N, Kajiyama K. Primary hepatic mucosa-associated lymphoid tissue lymphoma: a case report and literature review. Surg Case Rep. 2015;1:87.

8. Doi H, Horiike N, Hiraoka A, et al. Primary hepatic marginal zone B cell lymphoma of mucosa-associated lymphoid tissue type: case report and review of the literature. Int J Hematol. 2008;88(4):418-423.

9. Mehrain S, Schima W, Ba-Ssalamah A, et al. Primary MALT-lymphoma of the liver: multimodality imaging. Crit Rev Comput Tomogr. 2003;44(6):347-355.

10. Wotherspoon AC, Ortiz-Hidalgo C, Falzon MR, et al. Helicobacter pylori-associated gastritis and primary B-cell gastric lymphoma. Lancet. 1991;338(8776):1175-1176. 